\title{
Safety and efficacy of hysteroscopic sterilization compared with laparoscopic sterilization: an observational cohort study
}

\author{
Jialin Mao, ${ }^{1}$ Samantha Pfeifer, ${ }^{2}$ Peter Schlegel, ${ }^{3}$ Art Sedrakyan ${ }^{1}$
}

1Department of Health Policy and Research, Weill Medical College of Cornell University, New York, NY 10065, USA

2Department of Obstetrics and Gynecology, Weill Medical

College of Cornell University,

New York-Presbyterian Hospital, New York, NY, USA

3Department of Urology, Weill Medical College of Cornell

University, New York-

Presbyterian Hospital,

New York, NY, USA

Correspondence to:

A Sedrakyan

ars2013@med.cornell.edu

Additional material is published online only. To view please visit

the journal online (http://dx.doi. org/10.1136/bmj.h5162)

Cite this as: BMJ 2015;351:h5162 doi: 10.1136/bmj.h5162

Accepted: 23 September 2015

\section{ABSTRACT}

OBJECTIVE

To compare the safety and efficacy of hysteroscopic sterilization with the "Essure" device with laparoscopic sterilization in a large, all-inclusive, state cohort.

DESIGN

Population based cohort study.

SETTINGS

Outpatient interventional setting in New York State.

\section{PARTICIPANTS}

Women undergoing interval sterilization procedure, including hysteroscopic sterilization with Essure device and laparoscopic surgery, between 2005 and 2013.

\section{MAIN OUTCOMES MEASURES}

Safety events within 30 days of procedures; unintended pregnancies and reoperations within one year of procedures. Mixed model accounting for hospital clustering was used to compare 30 day and 1 year outcomes, adjusting for patient characteristics and other confounders. Time to reoperation was evaluated using frailty model for time to event analysis.

RESULTS

We identified 8048 patients undergoing hysteroscopic sterilization and 44278 undergoing laparoscopic sterilization between 2005 and 2013 in New York State. There was a significant increase in the use of hysteroscopic procedures during this period, while use of laparoscopic sterilization decreased. Patients undergoing hysteroscopic sterilization were older than those undergoing laparoscopic sterilization and were more likely to have a history of pelvic inflammatory disease $(10.3 \% \vee 7.2 \%, \mathrm{P}<0.01)$, major abdominal surgery $(9.4 \% \vee 7.9 \%, \mathrm{P}<0.01)$, and cesarean section $(23.2 \%$ v $15.4 \%, \mathrm{P}<0.01)$. At one year after surgery, hysteroscopic sterilization was not associated with a

\section{WHAT IS ALREADY KNOWN ON THIS TOPIC}

Laparoscopic bilateral tubal ligation has been the primary method of female permanent birth control for decades, and hysteroscopic microinsert device was developed as a less invasive alternative method

Since the procedure's approval, there has been thousands of reports of adverse events related to the use of hysteroscopic sterilization, but there is little information regarding its safety and efficacy compared with tubal ligation

\section{WHAT THIS STUDY ADDS}

This population based cohort study found that patients who underwent hysteroscopic sterilization did not have a higher risk of unintended pregnancy than those who underwent laparoscopic tubal ligation

However hysteroscopic sterilization was associated with over 10 -fold higher risk of reoperation, and the higher risk of reoperation persisted in various age groups and patients with history of pelvic inflammatory disease

higher risk of unintended pregnancy (odds ratio 0.84 ( $95 \% \mathrm{Cl} 0.63$ to 1.12)) but was associated with a substantially increased risk of reoperation (odds ratio 10.16 (7.47 to 13.81)) compared with laparoscopic sterilization.

\section{CONCLUSIONS}

Patients undergoing hysteroscopic sterilization have a similar risk of unintended pregnancy but a more than 10 -fold higher risk of undergoing reoperation compared with patients undergoing laparoscopic sterilization. Benefits and risks of both procedures should be discussed with patients for informed decisions making.

\section{Introduction}

Female sterilization is one of the most commonly used methods of contraception worldwide and is adopted by over 10 million women of reproductive age in the United States. ${ }^{1}$ Bilateral tubal ligation via laparoscopic approach or mini-laparotomy has been the primary technique for decades; and implant based sterilization by means of a hysteroscopic approach was developed as a less invasive alternative. The "Essure" device received approval in Europe (Conformité Européenne (CE) mark) in 2001 and was approved by the US Food and Drug Administration (FDA) in 2002. ${ }^{2}$ It is used in North America, Europe, Australia, New Zealand, Central and South America, and the Middle East. ${ }^{3}$

The hysteroscopic procedure with Essure device does not require general anesthesia, and its safety has been considered to be similar or superior to that of laparoscopic sterilization. ${ }^{4}$ However, the hysteroscopic approach was reported to be associated with a higher risk of unintended pregnancy and has a three month post-procedure waiting period before sterilization becomes effective. ${ }^{4}$ Unintended pregnancies can be considered as a failure of the procedure and can lead to a higher risk of potentially lethal ectopic pregnancies. ${ }^{5}$ Other reported complications related to device include pelvic pain, hemorrhage, and device migration or incompatibility $^{6}$ that can lead to reoperation. Since the Essure device's approval, thousands of reports of adverse events related to the device have been received by the FDA, and device failure became a subject of litigation in 2014..$^{6-8}$

The only prospective data regarding safety and efficacy of hysteroscopic sterilization was reported by phase II and phase III studies sponsored by the manufacturer. ${ }^{9-11}$ No randomized controlled trial or large comparative cohort study has been conducted to compare the efficacy and safety of the implant based hysteroscopic procedure with the traditional laparoscopic procedure. The purpose of our study was to evaluate the performance, safety, and other outcomes of hysteroscopic sterilization compared with laparoscopic 
sterilization using statewide and all-inclusive population data. Our secondary objective was to conduct subgroup analyses by age and important risk factors.

\section{Methods}

\section{Data source}

We used data from New York State Department of Health Statewide Planning and Research Cooperative System (SPARCS) to conduct an observational cohort study. Established in 1979, SPARCS is an all age group, all payer database that collects patient and treatment information for every hospital discharge, outpatient service, ambulatory surgery, and emergency department record in New York State. ${ }^{12}$ The data contains patient characteristics, primary and secondary diagnoses and procedures, length of stay, and charges. A unique personal identifier is assigned to every patient and encrypted to allow longitudinal analyses without compromising the confidentiality of the records.

\section{Study population}

Using CPT-4 (Current Procedural Terminology coding system, fourth edition) and ICD-9-CM (international classification of diseases, ninth revision, clinical modification) codes, we identified women undergoing hysteroscopic sterilization (CPT-4 58565) or laparoscopic sterilization (CPT-4 58670, 58671; ICD-9-CM 66.2x, 66.3x) with the encounter of sterilization (ICD-9-CM V25.2) between 2005 and 2013 in New York State. The CPT code for hysteroscopic sterilization was released at the end of 2004 and became available in SPARCS in 2005. We only included patients who underwent the procedure in an outpatient setting for interval sterilization for the purpose of valid comparison. Patients who underwent sterilization via laparotomy or concurrent with other abdominal procedures were excluded. We defined the first sterilization procedure available for each patient in SPARCS as the index procedure within the 2005-13 study period. Patients who had previously undergone sterilization procedures were excluded from the final cohort as those were not eligible cases to be considered as the index procedure. In total, 52326 women were included in the final analyses (see appendix A in supplementary data).

\section{Variable definitions}

Patient characteristics included age $(<30,30-39$, and $\geq 40$ years), race and ethnicity (non-Hispanic white, non-Hispanic black, Hispanic, and other), insurance status (Medicare, Medicaid, commercial, and other), comorbidities, previous pelvic inflammatory disease, history of major abdominal surgery, history of cesarean section, and anesthesia received during the procedure. Age was categorized based on previous description on sterilization patterns in the US. ${ }^{1}$ Major comorbidities were identified using algorithms validated by Elixhauser, ${ }^{13}$ they include coronary artery disease, hypertension, congestive heart failure, diabetes, chronic pulmonary disease, obesity, anemia, peripheral vascular disease, cerebrovascular disease, renal failure, and depression. Patients with pelvic adhesions, such as from previous surgery and pelvic inflammatory disease, traditionally were not considered good candidates for laparoscopic sterilization ${ }^{14}$ and have been shown to have higher risks of complications after surgery. ${ }^{1516}$ Therefore, we included prior history of pelvic inflammatory disease, major abdominal surgery, and cesarean section (from hospitalization and outpatient records) before their index surgery date since 1995 (the first year SPARCS data were available for our use). ICD-9 and CPT codes were used to identify history of pelvic inflammatory disease, major abdominal surgery, and cesarean section. History of pelvic inflammatory disease was determined based on any records identified from the database, including inpatient, outpatient, and emergency room settings.

Outcomes included procedure time and total charges; 30 day surgical safety; and one year follow-up for unintended pregnancy, ectopic pregnancy, and reoperation after the initial procedure. The 30 day surgical safety events included iatrogenic complications (hemorrhage or hematoma complicating a procedure and accidental puncture or laceration) and major medical complications (acute myocardial infarction, stroke, pulmonary embolism, perioperative shock, and respiratory complications). Pregnancy was identified from inpatient, emergency room and outpatient admission specifically for pregnancy related care, including prenatal tests and exams and care of ectopic or abortive pregnancy or other abnormal pregnancy after the index procedure. Patients who had a tubal reversal surgery or in vitro fertilization and became pregnant were not defined as unintended pregnancy. Reoperation was determined based on repeated sterilization procedures and identified using ICD-9 and CPT codes in subsequent records during follow-up. In the main analyses, follow-up was limited to one year to avoid loss of follow-up because of relocation of patients. Longer term analysis was conducted to evaluate unintended pregnancy and reoperation within two and three years. Detailed definition of outcome variables are listed in appendix B in the supplementary data.

\section{Statistical analyses}

Use of hysteroscopic sterilization and laparoscopic sterilization over time were inspected graphically and within age groups. Trend in use was assessed with Cochran-Armitage trend test. Baseline characteristics were compared between patients undergoing hysteroscopic and laparoscopic sterilization. Events and percentages were presented for patient demographics and comorbidities. In-hospital, 30 day, and one year outcomes were examined for each group. Differences per 1000 patients and 95\% confidence intervals were calculated for unadjusted categorical outcomes. Differences in median and interquartile ranges were obtained for continuous outcomes. We used $\chi^{2}$ tests for categorical variables and Wilcoxon rank sum tests for continuous variables to compare differences in baseline characteristics and unadjusted outcomes between groups.

Because of the structure of data-where patients were nested in institutions where procedures were performed-we used random effects models (generalized linear mixed model and frailty model) to account for 
variations between institutions..$^{17}$ A generalized linear mixed model, including hospital clustering as a random effect, was adopted to compare 30 day safety and one, two, and three year outcomes between the two groups. We adjusted the model for patient age, ethnicity, insurance status, procedure year, major comorbidities and history of pelvic inflammatory disease, major abdominal surgeries, and cesarean section. Time to reoperation was inspected graphically and evaluated using time to event analysis. A frailty model was used to account for hospital clustering in adjusted analysis.

Subgroup analysis was performed among patients of different age group and among patients with or without history of pelvic inflammatory disease respectively. A similar method was applied to subgroup analysis. All analyses were performed using SAS v9.3 (SAS Institute, Cary, NC). Statistical significance was defined as $\mathrm{P}<0.05$.

\section{Sensitivity analyses to address missing data}

We used three strategies to handle missing data: (a) exclusion of individuals with missing characteristics, (b) creation of a "missing" category, and (c) multiple imputation. We created a non-missing experimental sample by including only patients who had complete information. To improve reliability of our analyses, we partitioned data into a 70\% training sample and a 30\% validation sample. Using reoperation at one year as our outcome variable, estimates of odds ratio from the non-missing sample were regarded as the "true estimate." To simulate the situation, missing race information was assigned to $2 \%$ of patients and missing insurance payer information was assigned to $0.1 \%$ of patients randomly. Multiple imputation with logistic regression methods was developed and compared with exclusion of individuals with missing characteristics and with creation of a missing category to cope with missing data. The multiple imputation strategy developed was then validated on the $30 \%$ validation sample.

Estimates of odds ratios yielded by the three approaches were summarized and included in appendix C of the data supplement. Strategies $(b)$ and $(c)$ obtained similar results, with creation of a missing variable category being slightly more accurate when compared with the "true estimate," but the results were all comparable.

\section{Ethical approval}

This study was approved by the Weill Cornell Medical College Institutional Review Board (protocol number 1209013064).

\section{Patient involvement}

No patients were involved in setting the research question or the outcome measures, nor were they involved in the design and implementation of the study. There are no plans to involve patients in dissemination.

\section{Results}

In total, 8048 women underwent implant based hysteroscopic sterilization and 44278 underwent laparoscopic sterilization in outpatient setting between 2005 and 2013 in New York State. Over the study period, the

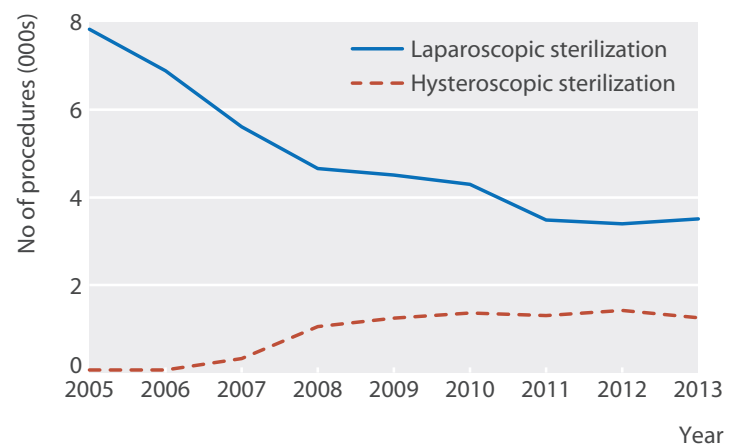

Fig 1 | Number of hysteroscopic sterilization and laparoscopic sterilization procedures between 2005 and 2013 in New York State

use of the two interval sterilization procedures decreased by $39.9 \%$ (from 7897 to 4748) (fig 1). However, hysteroscopic sterilization increased from 45 cases to 1231 cases, representing a rise from $0.6 \%$ to $25.9 \%$ of all cases $(\mathrm{P}<0.01)$. Use of laparoscopic procedure decreased from 7852 to 3517 over the same time. Use of hysteroscopic sterilization increased in all three age groups and was highest among women aged $\geq 40$ years (fig 2).

Most women undergoing sterilization procedures were in the age group 30-39 years (55.2\%) and had a commercial insurance $(55.9 \%)(\mathrm{P}<0.01)$ (table 1$)$. Compared with the patients undergoing laparoscopic sterilization, a larger proportion of patients undergoing hysteroscopic sterilization were $\geq 40$ years old $(25.2 \% v 20.5 \%, \mathrm{P}<0.01)$, had Medicaid coverage ( $43.6 \% v 37.1 \%, \mathrm{P}<0.01)$, and had one or more comorbidities $(19.7 \% v 13.0 \%, \mathrm{P}<0.01)$. Prevalence of previous pelvic inflammatory disease was also higher among patients undergoing hysteroscopic sterilization $(10.3 \% v 7.2 \%, \mathrm{P}<0.01)$. Patients in the hysteroscopic group were also more likely to have a history of major abdominal surgery $(9.4 \% v 7.9 \%, \mathrm{P}<0.01)$ or cesarean section $(23.2 \% v 15.4 \%, \mathrm{P}<0.01)$.

\section{Unadjusted analyses}

Surgical iatrogenic complications and major medical complications following both procedures were rare $(<0.5 \%)$ (table 2). Unintended pregnancy occurrence was similar after hysteroscopic sterilization and laparoscopic sterilization $(1.2 \% \vee 1.1 \%, \mathrm{P}=0.66)$. However, reoperation risk within one year was higher after

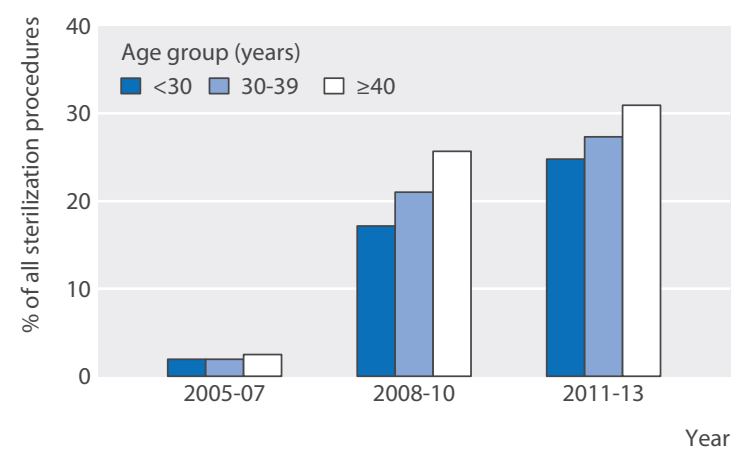

Fig 2 | Use of hysteroscopic sterilization by age group between 2005 and 2013 in New York State 


\begin{tabular}{|c|c|c|c|}
\hline & $\begin{array}{l}\text { Hysteroscopic } \\
\text { sterilization } \\
(\mathrm{n}=8048)\end{array}$ & $\begin{array}{l}\text { Laparoscopic } \\
\text { sterilization } \\
(n=44278)\end{array}$ & $\begin{array}{l}\text { P value of } \\
\text { difference }\end{array}$ \\
\hline \multicolumn{3}{|l|}{ Age (years): } & \multirow{4}{*}{$<0.01$} \\
\hline$<30$ & $1601(19.9)$ & $10715(24.2)$ & \\
\hline $30-39$ & $4416(54.9)$ & $24484(55.3)$ & \\
\hline$\geq 40$ & $2031(25.2)$ & $9079(20.5)$ & \\
\hline \multicolumn{3}{|l|}{ Ethnicity*: } & \multirow{5}{*}{$<0.01$} \\
\hline White (non-Hispanic) & $3871(48.5)$ & $23289(54.0)$ & \\
\hline Black (non-Hispanic) & $1400(17.5)$ & $6283(14.6)$ & \\
\hline Hispanic & $1760(22.0)$ & $9273(21.5)$ & \\
\hline Other & $957(12.0)$ & $4298(10.0)$ & \\
\hline \multicolumn{3}{|l|}{ Medical insurancet: } & \multirow{5}{*}{$<0.01$} \\
\hline Medicare & $166(2.1)$ & $796(1.8)$ & \\
\hline Medicaid & $3508(43.6)$ & $16398(37.1)$ & \\
\hline Commercial & $4005(49.8)$ & $25218(57.0)$ & \\
\hline Other & $368(4.6)$ & $1821(4.1)$ & \\
\hline \multicolumn{3}{|l|}{ No of major comorbidities: } & \multirow{4}{*}{$<0.01$} \\
\hline 0 & $6467(80.4)$ & $38551(87.1)$ & \\
\hline 1 & $1197(14.9)$ & $4725(10.7)$ & \\
\hline$\geq 2$ & $384(4.8)$ & $1002(2.3)$ & \\
\hline History of pelvic inflammatory disease & $832(10.3)$ & $3166(7.2)$ & $<0.01$ \\
\hline History of major abdominal surgery & $757(9.4)$ & $3519(7.9)$ & $<0.01$ \\
\hline History of cesarean section & $1867(23.2)$ & $6797(15.4)$ & $<0.01$ \\
\hline
\end{tabular}

hysteroscopic sterilization procedure $(2.4 \% \vee 0.2 \%$, $\mathrm{P}<0.01)$. Hysteroscopic procedures were associated with shorter procedure time (median $36 \mathrm{~min} v 52 \mathrm{~min}$, $\mathrm{P}<0.01)$, less frequent use of general anesthesia $(50.6 \%$ $v 75.8 \%, \mathrm{P}<0.01$ ), but higher total charges (median $\$ 7832 v \$ 5068, \mathrm{P}<0.01)$.

\section{Adjusted analyses}

After adjusting for patient characteristics and hospital clustering, we found hysteroscopic sterilization was associated with lower risk of iatrogenic complications within 30 days after surgery compared with laparoscopic sterilization (odds ratio 0.35 (95\% confidence interval 0.20 to 0.61)) (table 3). Hysteroscopic sterilization was not associated with a higher risk of unintended pregnancy (odds ratio 0.84 (0.63 to 1.12)). However, the odds of reoperation were much higher after hysteroscopic sterilization compared with laparoscopic sterilization (odds ratio 10.16 (7.47 to 13.81)).

Using time to event analysis for one year reoperation, we found the average time to reoperation was 20.0 and 22.9 weeks for women undergoing hysteroscopic sterilization and laparoscopic sterilization respectively (appendix D in data supplement). The risk of undergoing reoperation within one year after the initial procedure was again more than 10 times higher among women who underwent hysteroscopic sterilization with an implant (hazard ratio 10.13 (7.50 to 13.68)).

\section{Outcomes at two and three years after the procedure}

In these analyses, hysteroscopic sterilization was consistently associated with higher odds of reoperation but similar odds of pregnancy compared with laparoscopic sterilization (appendix E in data supplement). Patients undergoing hysteroscopic sterilization were eight times more likely to undergo a reoperation at two years after initial surgery (odds ratio 7.96 (6.00 to 10.57)) and six times more likely at three years (odds ratio 5.88 (4.44 to 7.79)).

\section{Risk factors and subgroup effects}

Among other risk factors, younger age was associated with higher risk of pregnancy but not reoperation after the procedures (table 3). Having a history of pelvic inflammatory disease was related to increased risks of 30 day safety events as well as one year pregnancy and reoperation. A history of pelvic inflammatory disease was associated with four times higher odds of unintended pregnancy (odds ratio 3.72 (3.00 to 4.59)) and two times higher odds of undergoing reoperation (odds ratio 1.67 (1.16 to 2.41)).

In subgroup analyses, hysteroscopic sterilization was associated with higher odds of reoperation but not of pregnancy compared with laparoscopic sterilization across all age groups (table 4). Within the group of

Table 2 | Procedure related data, unadjusted 30 day safety, and one year outcomes after hysteroscopic and laparoscopic sterilization between 2005 and 2013 in New York State. Values are numbers (percentages) of patients unless stated otherwise

\begin{tabular}{|c|c|c|c|c|}
\hline & Hysteroscopic sterilization & Laparoscopic sterilization & Difference $(95 \% \mathrm{Cl}) *$ & P value \\
\hline In hospital & $(n=8048)$ & $(n=44278)$ & & \\
\hline General anesthesia & $4072(50.6)$ & $33551(75.8)$ & $-252(-263$ to -240$)$ & $<0.01$ \\
\hline Median (IQR) procedure time (minutes) & $36(22$ to 52$)$ & $52(35$ to 70$)$ & $-16(-18$ to -13$)$ & $<0.01 \dagger$ \\
\hline Median (IQR) total charges $(\$)$ & $7832(5636$ to 10039$)$ & 5068 (3622 to 6996) & 2764 (2014 to 3043$)$ & $<0.01 \dagger$ \\
\hline 30 day follow-up $¥$ & $(n=7969)$ & $(n=44014)$ & & \\
\hline Iatrogenic complications & $14(0.2)$ & $188(0.4)$ & $-3(-4$ to -1$)$ & $<0.01$ \\
\hline Major medical complications & NR & $35(0.1)$ & NR & 0.90 \\
\hline One year follow-up $¥$ & $(n=6817)$ & $(n=40761)$ & & \\
\hline Pregnancy & $79(1.2)$ & $448(1.1)$ & $1(-3$ to 4$)$ & 0.66 \\
\hline Ectopic pregnancy & NR & $59(0.1)$ & NR & 0.03 \\
\hline Reoperation & $162(2.4)$ & $99(0.2)$ & 21 (18 to 25$)$ & $<0.01$ \\
\hline
\end{tabular}


Table 3 | Adjusted 30 day safety and one year outcomes after hysteroscopic and laparoscopic sterilization between 2005 and 2013 in New York State. Values are odds ratios ( $95 \%$ confidence intervals)

\begin{tabular}{|c|c|c|c|c|}
\hline & \multirow{2}{*}{$\begin{array}{l}\text { Hysteroscopic } v \text { laparoscopic } \\
\text { sterilization }\end{array}$} & \multicolumn{2}{|l|}{ Ages (years) } & \multirow{2}{*}{$\begin{array}{l}\text { History } v \text { no history of pelvic } \\
\text { inflammatory disease }\end{array}$} \\
\hline & & $<30 v \geq 40$ & $30-39 v \geq 40$ & \\
\hline \multicolumn{5}{|l|}{30 day follow-up* } \\
\hline latrogenic complications & $0.35(0.20$ to 0.61$)$ & 1.61 (1.02 to 2.54$)$ & 1.17 (0.77 to 1.77$)$ & 1.52 (0.98 to 2.35$)$ \\
\hline Major medical complications & 0.70 (0.28 to 1.78$)$ & $0.46(0.17$ to 1.23$)$ & $0.68(0.32$ to 1.46$)$ & $1.87(0.84$ to 4.17$)$ \\
\hline \multicolumn{5}{|l|}{ One year follow-upt } \\
\hline Pregnancy & 0.84 (0.63 to 1.12$)$ & $1.62(1.13$ to 2.33$)$ & 1.83 (1.32 to 2.53$)$ & $3.72(3.00$ to 4.59$)$ \\
\hline Ectopic pregnancy & $0.34(0.10$ to 1.13$)$ & 3.49 (1.00 to 12.19$)$ & 3.51 (1.07 to 11.58$)$ & 2.89 (1.59 to 5.25$)$ \\
\hline Reoperation & $10.16(7.47$ to 13.81$)$ & $1.06(0.70$ to 1.59$)$ & 1.17 (0.84 to 1.63$)$ & 1.67 (1.16 to 2.41$)$ \\
\hline
\end{tabular}

*Patients who received the procedure during the last month of 2013 were excluded for 30 day follow-up.

tPatients who received the procedure in 2013 were excluded for one year follow-up.

Model accounted for hospital clustering and adjusted for patient age, race, insurance status, year of procedure, major comorbidities, and history of pelvic inflammatory disease, major abdominal surgeries, and cesarean section.

patients with a history of pelvic inflammatory disease, the odds of pregnancy were lower after hysteroscopic sterilization compared with laparoscopic sterilization (odds ratio 0.54 (0.32 to 0.89$)$ ), but hysteroscopic sterilization was again associated with a higher odds of reoperation (odds ratio 4.10 (1.99 to 8.45)).

\section{Discussion}

In our study, we found an over 10-fold higher risk of reoperation associated with hysteroscopic sterilization with the Essure device compared to laparoscopic sterilization, translating into about 21 additional reoperations per 1000 patients undergoing surgery. Meanwhile, the occurrence of unintended pregnancy was not different in the two groups. We also found that the use of device based hysteroscopic sterilization increased from $0.6 \%$ of all surgeries in 2005 to $25.9 \%$ in 2013 and was more often performed in older patients and those with higher comorbidity compared with laparoscopic tubal ligation.

\section{Comparison with other studies}

Since the publication of multicenter cohort study including 9475 patients over 15 years ago, laparoscopic tubal ligation has been considered safe, with only rare occurrence of severe morbidity. ${ }^{15}$ Recently, hysteroscopic sterilization was also reported to be relatively safe in a review study that evaluated various complications. ${ }^{18}$ However, the comparative safety of laparoscopic tubal ligation and device based hysteroscopic sterilization procedure has not been studied. To the best of our knowledge, our study is the first population cohort based comparative investigation of safety and efficacy of two widely used procedures for permanent birth control. We confirmed that initial hysteroscopic and laparoscopic sterilization procedures are relatively safe, but charges for hysteroscopic sterilization were higher despite shorter operative time, similar occurrence of complications, and less frequent use of general anesthesia. Future studies with more detailed information on charges are needed to better understand whether charges translate into higher costs for hysteroscopic surgery. General anesthesia was less frequently used when performing hysteroscopic sterilization compared with laparoscopic sterilization but it was still used in about half of the patients. This finding is remarkable in light of the marketing and proposed benefits of avoiding general anesthesia associated with the Essure device.

The risks of experiencing unintended pregnancy after hysteroscopic and laparoscopic sterilization were higher in our study than those reported in previous studies. The US Collaborative Review of Sterilization (CREST) study in the 1990s reported that the cumulative 10 year probability of pregnancy following tubal ligation was $1.3 \% .^{19}$ The risk of unintended pregnancy following tubal ligation was believed to be lower than $1 \%$ during the first year after procedure. ${ }^{20}$ Meanwhile,

Table 4 | Unadjusted events and adjusted odds ratios of one year outcomes after hysteroscopic and laparoscopic sterilization stratified be age and history of pelvic inflammatory disease between 2005 and 2013 in New York State. Values are numbers (percentages) of patients unless stated otherwise

\begin{tabular}{|c|c|c|c|c|c|c|}
\hline & \multicolumn{3}{|c|}{ Unintended pregnancy } & \multicolumn{3}{|l|}{ Reoperation } \\
\hline & $\begin{array}{l}\text { Hysteroscopic } \\
\text { sterilization }\end{array}$ & $\begin{array}{l}\text { Laparoscopic } \\
\text { sterilization }\end{array}$ & $\begin{array}{l}\text { Comparison: odds } \\
\text { ratio }(95 \% \mathrm{Cl})^{*}\end{array}$ & $\begin{array}{l}\text { Hysteroscopic } \\
\text { sterilization }\end{array}$ & $\begin{array}{l}\text { Laparoscopic } \\
\text { sterilization }\end{array}$ & $\begin{array}{l}\text { Comparison: odds } \\
\text { ratio }(95 \% \mathrm{Cl})^{*}\end{array}$ \\
\hline \multicolumn{7}{|c|}{ Age group (years)t: } \\
\hline$<30$ & $23(1.7)$ & $113(1.1)$ & 1.09 (0.62 to 1.90$)$ & $36(2.7)$ & $22(0.2)$ & 11.06 (5.93 to 20.61) \\
\hline $30-39$ & $50(1.3)$ & $293(1.3)$ & 0.76 (0.53 to 1.09$)$ & $90(2.4)$ & $63(0.3)$ & 8.83 (5.98 to 13.04$)$ \\
\hline$\geq 40$ & NR & $42(0.5)$ & 0.47 (0.19 to 1.19 ) & $36(2.0)$ & $14(0.2)$ & 14.53 (6.55 to 32.21$)$ \\
\hline \multicolumn{7}{|c|}{ History of pelvic inflammatory diseaseł: } \\
\hline Yes & $26(4.0)$ & $172(6.3)$ & $0.54(0.32$ to 0.89$)$ & $18(2.8)$ & $21(0.8)$ & $4.10(1.99$ to 8.45$)$ \\
\hline No & $53(0.9)$ & $276(0.7)$ & 0.97 (0.69 to 1.36$)$ & $144(2.3)$ & $78(0.2)$ & 12.52 (8.85 to 17.72$)$ \\
\hline
\end{tabular}

NR=Not reportable for events fewer than 10 .

* Laparoscopic group is the reference group for comparisons.

tAdjustments in age stratified analyses accounted for hospital clustering, patient ethnicity, insurance status, procedure year, and major comorbidities including history of pelvic inflammatory disease, major abdominal surgeries, and cesarean section.

¥Adjustments in analyses stratified for pelvic inflammatory disease history accounted for hospital clustering, patient age, ethnicity, insurance status, procedure year, and major comorbidities

including history of major abdominal surgeries and cesarean section. 
hysteroscopic sterilization was shown to be $99.83 \%$ effective based on a five year clinical study. ${ }^{6}$ The newly released long term follow-up of a phase III study observed no pregnancy following hysteroscopic sterilization. ${ }^{11}$ A retrospective study from France conducted from 2006 to 2010 reported pregnancy rates of $0.36 \%$ and $0.46 \%$ after hysteroscopic and laparoscopic sterilization procedures respectively. ${ }^{21}$

Several factors may have contributed to the difference between our findings and prior evidence on unintended pregnancies. The published data were mostly based on highly selected clinical investigations or small studies. Clinical trials are conducted in a way different from real world settings and usually include only patients who are the best candidates for the procedure. ${ }^{22}$ For example, patients with history of pelvic inflammatory disease were not well represented in prior investigations. ${ }^{9}$ We conducted subgroup analyses of pelvic inflammatory disease patients and found significantly higher risk of pregnancy in this subgroup following both procedures. In addition, single or multicenter studies were usually conducted at selected high volume medical facilities, which perform better than most facilities in the entire state or country. The study from France was a population based study, ${ }^{21}$ but it captured pregnancy only through discharge records of hospitalization for pregnancy or delivery. This may lead to underestimation of risk of unintended pregnancy following procedures, with the omission of miscarriage or abortion not requiring hospitalization. Our study was able to identify prenatal tests following sterilization and provide more accurate estimates. In addition, sterilization is indicated for women aged over 40 in France, ${ }^{21}$ who had lower probability of pregnancy. In our subgroup analyses of women aged over 40 years, pregnancy risk was similar to that reported in the French cohort.

\section{Risk of reoperation}

Reoperation occurrence is an important patient centered outcome in surgery. It has been used as an indicator of surgical and device performance in high quality studies in various surgical topics including orthopedics, general surgery, and urology. ${ }^{23-25}$ A more than 10 -fold higher occurrence of reoperation during the first year following Essure based surgery is a serious safety concern. While reoperation following sterilization procedure can be related to unintended pregnancy, the similar risk of unintended pregnancy for both procedures in our study indicated that additional surgeries were performed to alleviate complications such as device migration or incompatibility after surgery. ${ }^{26}$ According to our review of the Manufacturer and User Facility Device Experience (MAUDE) database, ${ }^{27}$ most of the adverse events reported by patients for the Essure based procedure were injuries that would require a reoperation (see appendix $\mathrm{F}$ in data supplement).

Some of the reoperations after Essure use might potentially be related to "detection bias," as follow-up care is generally required within three months after hysteroscopic sterilization. ${ }^{28}$ These follow-up procedures may have helped to detect and manage early device failures, contributing to the higher reoperation risk found in our study. However, the three month follow-up requirement may have also favored the Essure based procedure in managing patients and not raising the risk of hospital admission with unintended pregnancy.

\section{Strengths and limitations of study}

There are a few limitations in our study using New York State data. Since patients are not likely to be hospitalized or go to emergency room for pelvic pain or changes in menstrual cycle, we were unable to investigate risk of developing pelvic pain or having prolonged menstrual cycle following the procedures. Our outcome of interest was reoperation (repeated sterilization) procedure. We were unable to identify women who did not undergo procedures to manage device related complications or if reoperation was not coded as another sterilization procedure. Hence, we included an appendix with information from the MAUDE database to provide relevant information on other safety events that might not require reoperation. Loss of follow-up may occur as a consequence of relocation of patients to another state or country in two and three year follow-up periods. However, it is unlikely to differentially affect the hysteroscopic and laparoscopic groups. In addition, inherent with administrative databases, identification of comorbidities and outcomes relies on diagnosis and procedure coding. It has been shown to be mostly appropriate but minor coding errors may still occur. ${ }^{29}$

We obtained patient histories of pelvic inflammatory disease, major abdominal surgery, and cesarean section from patients' medical records, some under-coding was possible, potentially leading to residual confounding. However, it is unlikely that this will have a strong impact on our estimates considering adjusted analyses, statistical significance, and the magnitude of the association. Some misclassification of Essure is also possible during the early years of the CPT code's introduction. With Essure procedure misclassified as laparoscopic procedure, however, the bias would be toward null and the real risk of reoperation following hysteroscopic sterilization compared with laparoscopic sterilization would be higher.

\section{Study implications}

In our study using entire New York state all-payer data, we are able to provide evidence that is generalizable to common patient population. This study is aligned with the Idea, Development, Exploration, Assessment, Longterm follow-up (IDEAL) principles that highlights the need of technology surveillance after widespread adoption. ${ }^{30}$ We illustrate that large, statewide, "real world" population cohort studies enable evaluation of device or surgery performance after wide adoption. These data, along with national and regional registries, are key tools for long term evaluation of surgical techniques and device technologies. ${ }^{30}$ With an estimated 600000 sterilization procedures performed in the US every year, ${ }^{31}$ the device based hysteroscopic sterilization has a major public health impact, and comparative safety and effectiveness of sterilizations are important for decision making by patients and physicians. A registry based 
study with longer follow-up is warranted to further understand the failure events after device use and improve the safety and efficacy of sterilization procedures.

\section{Conclusions}

Patients undergoing hysteroscopic sterilization have a similar risk of unintended pregnancy but more than 10-fold higher risk of undergoing a reoperation when compared with laparoscopic sterilization. Benefits and risks of both procedures should be discussed with patients for informed decisions making.

We thank Madris Tomes, developer of a post-market surveillance MAUDE analytics tool for providing up to date information about safety reports related to Essure.

Contributors: JM and AS were responsible for the study concept and design. AS acquired the data. JM and AS analyzed and all authors interpreted the data. All authors drafted the manuscript and critically revised it for important intellectual content. JM and AS were responsible for the statistical analysis. AS supervised the study and is the guarantor.

Funding: The study was funded in part through U01 grant $(\mathrm{NIH}-$ 1U01FD004494-01) from the National Institutes of Health and US Food and Drug Administration. AS received the funding for establishing the MDEpiNet Science and Infrastructure Centre. JM is an analyst within the Weill Cornell Medical College (WCMC) Patient Centered Comparative Effectiveness Program and the Medical Device Epidemiology Network's (MDEpiNet) Science and Infrastructure Center: AS is the director of the Center).

Competing interests: All authors have completed the ICMJE uniform disclosure form at www.icmje.org/coi_disclosure.pdf (available on request from the corresponding author) and declare: no support from any organisation for the submitted work (other than the funding listed); no financial relationships with any organisations that might have an interest in the submitted work in the previous three years; no other relationships or activities that could appear to have influenced the submitted work.

Ethical approval: The study was approved by the Weill Cornell Medical College institutional review board (protocol no 1209013064).

Transparency: The lead authors (study guarantors) affirm that this manuscript is an honest, accurate, and transparent account of the study being reported; that no important aspects of the study have been omitted; and that any discrepancies from the study as planned (and, if relevant, registered) have been explained.

Data sharing: The technical appendix and statistical code available from the corresponding author at ars2013@med.cornell.edu. The dataset is available from New York State Department of Health Statewide Planning and Research Cooperative System (SPARCS) at https://www.health.ny.gov/statistics/sparcs/access/

This is an Open Access article distributed in accordance with the Creative Commons Attribution Non Commercial (CC BY-NC 4.0) license, which permits others to distribute, remix, adapt, build upon this work non-commercially, and license their derivative works on different terms, provided the original work is properly cited and the use is non-commercial. See: http://creativecommons.org/licenses/ by-nc/4.0/.

1 Jones J, Mosher W, Daniels K. Current contraceptive use in the United States, 2006-2010, and changes in patterns of use since 1995. Natl Health Stat Report 2012;(60):1-25.

2 US Food and Drug Administration. Essure system-P020014. 2002. www.fda.gov/MedicalDevices/ProductsandMedicalProcedures/ DeviceApprovalsandClearances/Recently-ApprovedDevices/ ucm083087.htm.

3 GlobeNewswire. Conceptus $(R)$ receives CE mark for transvaginal ultrasound confirmation for Essure(R) procedure. 2011. http:// globenewswire.com/news-release/2011/02/24/440957/214723/en/ Conceptus-R-Receives-CE-Mark-for-Transvaginal-UltrasoundConfirmation-for-Essure-R-Procedure.html.

4 Hurskainen R, Hovi SL, Gissler M, et al. Hysteroscopic tubal sterilization: a systematic review of the Essure system. Fertil Steril 2010;94:16-9.

5 Peterson HB, Xia Z, Hughes JM, Wilcox LS, Tylor LR, Trussell J. The risk of ectopic pregnancy after tubal sterilization. US Collaborative Review of Sterilization Working Group. N Engl I Med 1997;336:762-7.

6 US Food and Drug Administration. Essure permanent birth control. 2015. www.fda.gov/MedicalDevices/ProductsandMedicalProcedures/ ImplantsandProsthetics/ucm371014.htm.
7 Gilger L, Tomasch M. First lawsuit filed against FDA-approved Essure permanent birth control, Bayer HealthCare. ABC15, 2014. www.abc15. $\mathrm{com} /$ news/local-news/investigations/first-lawsuit-filed-against-fda-approvedessure-permanent-birth-control-bayer-healthcare.

8 Rabin RC. Long-term data on complications adds to criticism of Essure contraceptive implant. New York Times 2015 May 3. www.nytimes. com/2015/05/04/us/long-term-data-on-complications-adds-tocriticism-of-contraceptive-implant.html?_r=0.

9 Kerin JF, Cooper JM, Price T, et al. Hysteroscopic sterilization using a micro-insert device: results of a multicentre Phase II study. Hum Reprod 2003;18:1223-30.

10 Cooper JM, Carignan CS, Cher D, Kerin JF. Selective Tubal Occlusion Procedure Investigators G. Microinsert nonincisional hysteroscopic sterilization. Obstet Gynecol 2003;102:59-67.

11 ChudnoffSG, Nichols JE Jr, Levie M. Hysteroscopic Essure inserts for permanent contraception-extended follow-up results of a phase III, multicenter, international study. J Minim Invasive Gynecol 2015;22:951-60.

12 New York State Department of Health;. Statewide Planning and Research Cooperative System (SPARCS). 2014. www.health.ny.gov/ statistics/sparcs/.

13 Elixhauser A, Steiner C, Harris DR, Coffey RM. Comorbidity measures for use with administrative data. Med Care 1998;36:8-27.

14 Szigetvari I, Feinman M, Barad D, Bartfai G, Kaali SG. Association of previous abdominal surgery and significant adhesions in laparoscopic sterilization patients. J Reprod Med 1989;34:465-6.

15 Jamieson DJ, Hillis SD, Duerr A, Marchbanks PA, Costello C, Peterson HB. Complications of interval laparoscopic tubal sterilization: finding from the United States Collaborative Review of Sterilization. Obstet Gynecol 2000;96:997-1002.

16 Destefano F, Greenspan JR, Dicker RC, Peterson HB, Strauss LT, Rubin GL. Complications of interval laparoscopic tubal sterilization. Obstet Gynecol 1983;61:153-8.

17 Rice N, Leyland A. Multilevel models: applications to health data. J Health Serv Res Policy 1996;1:154-64.

18 Ouzounelli M, Reaven NL. Essure hysteroscopic sterilization versus interval laparoscopic bilateral tubal ligation: a comparative effectiveness review. I Minim Invasive Gynecol 2015;22:342-52.

19 Peterson HB, Xia Z, Hughes JM, Wilcox LS, Tylor LR, Trussell J. The risk of pregnancy after tubal sterilization: findings from the US Collaborative Review of Sterilization. Am J Obstet Gynecol 1996;174:1161-8.

20 Gariepy AM, Creinin MD, Smith KJ, Xu X. Probability of pregnancy after sterilization: a comparison of hysteroscopic versus laparoscopic sterilization. Contraception 2014;90:174-81.

21 Fernandez H, Legendre G, Blein C, Lamarsalle L, Panel P. Tubal sterilization: pregnancy rates after hysteroscopic versus laparoscopic sterilization in France, 2006-2010. Eur J Obstet Gynecol Reprod Biol 2014;180:133-7.

22 Sedrakyan A, Marinac-Dabic D, Normand SL, Mushlin A, Gross T. A framework for evidence evaluation and methodological issues in implantable device studies. Med Care 2010;48(6 suppl):S121-8.

23 Makela KT, Matilainen M, Pulkkinen P, et al. Failure rate of cemented and uncemented total hip replacements: register study of combined Nordic database of four nations. BMJ 2014;348:f7592.

24 Burns EM, Bottle A, Aylin P, Darzi A, Nicholls RJ, Faiz O. Variation in reoperation after colorectal surgery in England as an indicator of surgical performance: retrospective analysis of hospital episode statistics. BMJ 2011;343:d4836.

25 Chughtai B, Mao J, Buck J, Kaplan S, Sedrakyan A. Use and risks of surgical mesh for pelvic organ prolapse surgery in women in New York state: population based cohort study. BMJ 2015;350:h2685.

26 Connor VF. Essure: a review six years later. J Minim Invasive Gynecol 2009;16:282-90.

27 US Food and Drug Administration. MAUDE - manufacturer and use facility device experience. 2015. https://www.accessdata.fda.gov/ scripts/cdrh/cfdocs/cfmaude/search.cfm.

28 Cleary TP, Tepper NK, Cwiak C, et al. Pregnancies after hysteroscopic sterilization: a systematic review. Contraception 2013;87:539-48.

29 O'Malley KJ, Cook KF, Price MD, Wildes KR, Hurdle JF, Ashton CM. Measuring diagnoses: ICD code accuracy. Health Serv Res 2005;40:1620-39

30 Cook JA, McCulloch P, Blazeby JM, et al. IDEAL framework for surgical innovation 3: randomised controlled trials in the assessment stage and evaluations in the long term study stage. BMJ 2013;346:f2820.

31 American College of Obstetricians and Gynecologists. ACOG Practice bulletin no. 133: benefits and risks of sterilization. Obstet Gynecol 2013;121:392-404

(C) BMJ Publishing Group Ltd 2015

Appendices: A-Patient selection process, $\mathrm{B}-$ Definition of outcomes, $\mathrm{C}$-Comparison of methods in missing data handling, D-Time to event analysis of reoperation within 1 year following procedure, $\mathrm{E}-$ Long term follow-up of patients, F-MAUDE reports of adverse events related to Essure placement. 